\title{
Perfil epidemiológico dos acidentes de trabalho graves no estado da Bahia entre os anos de 2007 e 2017
}

\author{
Epidemiological profile of serious work accidents in the state of Bahia between the years \\ of 2007 and 2017
} Perfil epidemiológico de accidentes de trabajo graves en el estado de Bahia entre 2007 y
2017

\author{
Maria Inês Pardo Calazans ${ }^{1 *}$, Adriana Alves Nery².
}

\begin{abstract}
RESUMO
Objetivo: Descrever o perfil epidemiológico dos acidentes de trabalho graves notificados no estado da Bahia, entre 2007 a 2017. Métodos: Estudo ecológico, de natureza descritiva, onde foram obtidos dados das fichas de investigação epidemiológica. A unidade de observação dos dados foi apresentada de acordo com as nove macrorregiões de saúde do estado. A análise baseada na estatística descritiva. Resultados: Foram notificados 20.345 acidentes graves no período em questão; observou-se um incremento de $93 \%$ na incidência de casos, com frequência maior nos homens jovens, cor parda, ensino fundamental incompleto. Grande subnotificação na variável que identifica as atividades econômicas e quanto à ocupação, a categoria de produção de bens e serviços I, foi a mais representativa. Predominaram os acidentes típicos, sendo as instalações do contratante apontadas como local do acidente mais frequente. Observada grande subnotificação para a Comunicação de Acidente de Trabalho (40,5\%), sendo $91 \%$ das notificações com necessidade de atendimento médico. Conclusão: Mesmo diante às limitações que esse sistema de informação apresenta, observou-se que os acidentes graves representam um fenômeno de incidência expressiva em todo estado. Tais achados requerem atenção dos gestores, nas ações voltadas à melhoria das condições de trabalho e saúde, maior compromisso das instituições empregadoras e classe trabalhadora.
\end{abstract}

Palavras-chave: Acidentes de trabalho graves, Notificação de acidentes de trabalho, Vigilância em saúde do trabalhador.

\begin{abstract}
Objective: To describe the epidemiological profile of serious work accidents reported in the state of Bahia, between 2007 and 2017. Methods: Ecological study of descriptive nature, where data from epidemiological investigation records were obtained. The data observation unit was presented according to the nine health macroregions of the state. Analysis based on descriptive statistics. Results: A total of 20,345 serious accidents were reported in the period in question; there was a $93 \%$ increase in the incidence of cases, with higher frequency in young men, brown color, incomplete elementary school. Great underreporting in the variable that identifies economic activities and occupation, the production category of goods and services I was the most representative. Typical accidents predominated, and the contractor's facilities were identified as the most frequent accident site. There was a great underreporting for the Communication of Work Accident (40.5\%), and $91 \%$ of the notifications requiring medical care. Conclusion: Even in view of the limitations that this information system presents, it was observed that serious accidents represent a phenomenon of significant incidence throughout the state. These findings require attention from managers, in actions aimed at improving working conditions and health, greater commitment of employers and working class.
\end{abstract}

Keywords: Serious work accidents, Notification of accidents at work, Occupational health surveillance.

${ }^{1}$ Universidade Estadual do Sudoeste da Bahia (UESB), Jequié - BA. *E-mail: inezcalazans@gmail.com 
RESUMEN

Objetivo: Describir el perfil epidemiológico de los accidentes de trabajo graves reportados en el estado de Bahía, entre 2007 y 2017. Métodos: Estudio ecológico de carácter descriptivo, donde se obtuvieron datos de registros de investigación epidemiológica. La unidad de observación de datos se presentó de acuerdo con las nueve macrorregiones de salud del estado. Análisis basado en estadísticas descriptivas. Resultados: En el período en cuestión se notificaron un total de 20.345 accidentes graves; hubo un aumento del $93 \%$ en la incidencia de casos, con mayor frecuencia en hombres jóvenes, color marrón, escuela primaria incompleta. Gran subinformación en la variable que identifica las actividades económicas y la ocupación, la categoría de producción de bienes y servicios que fui el más representativo. Predominaron los accidentes típicos, y las instalaciones del contratista fueron identificadas como el lugar de accidentes más frecuentes. Hubo una gran información insuficiente para la Comunicación de Accidentes de Trabajo $(40,5 \%)$ y el $91 \%$ de las notificaciones que requerían atención médica. Conclusión: Incluso en vista de las limitaciones que presenta este sistema de información, se observó que los accidentes graves representan un fenómeno de incidencia significativa en todo el estado. Estos hallazgos requieren la atención de los gerentes, en acciones encaminadas a mejorar las condiciones de trabajo y la salud, un mayor compromiso de los empleadores y la clase trabajadora.

Palabras clave: Accidentes de trabajo graves, Notificación de accidentes de trabajo, Vigilancia de la salud ocupacional.

\section{INTRODUÇÃO}

Dos agravos relacionados ao trabalho, os Acidentes de Trabalho Graves (ATG) são eventos que tem propiciado o aumento da morbimortalidade no Brasil, nas últimas três décadas, configurando como um problema de saúde pública, pelas implicações que trazem aos trabalhadores, levando à redução da capacidade para o trabalho e morte, onerando as empresas e o Estado, mais especificamente a Previdência Social, através de benefícios e aposentadoria precoce, além dos gastos com internações hospitalares e reabilitação (CAVALCANTE CAA, et al., 2015; SCUSSIATO LA, et al., 2013).

Os ATG são definidos como eventos que acontecem no exercício da atividade laboral, bem como os que ocorrem no trajeto da residência para o trabalho ou vice-versa, não importando o vínculo de trabalho, acarretando na redução ou perda da capacidade para o trabalho, resultando em lesão corporal grave (temporária ou permanente) e/ou levar à morte. Também são entendidos como acidentes graves os que ocorrem com menores de dezoito anos (BAHIA, 2009; BRASIL, 2004; MIRANDA FMD, et al., 2012).

Segundo o Observatório Digital de Saúde e Segurança do Trabalho (2018), ocorreram no Brasil, entre os anos de 2012 e 2018, aproximadamente 16.500 mortes acidentárias notificadas, sendo uma morte a cada três horas.

O estado da Bahia tem apresentado um aumento preocupante dos ATG. No período de 2007 a 2012 , foram 5.771 notificações, evidenciando um aumento de 5 vezes no número dos registros, passando de 5,8\% em 2007 para 29\% em 2012 (SILVA AR, 2017). Entretanto, é importante ressaltar que esse aumento pode ter relação com a melhoria dos registros nos sistemas de informação em saúde.

Como são eventos considerados de notificação compulsória imediata e investigação obrigatória, os ATG devem ser notificados através da Comunicação de Acidente de Trabalho (CAT) quando o trabalhador for celetista. Nos casos de trabalhadores que pertençam a outros tipos de vínculos, o evento deverá ser 'comunicado às autoridades sanitárias locais, sejam por serviços públicos ou privados. As notificações são realizadas pelo preenchimento de ficha específica para ATG e registradas no Sistema de Informações de Agravos de Notificação (SINAN) (BAHIA, 2016; BRASIL, 2016).

O SINAN abrange uma extensa fonte de registros de notificação, inclusive dos eventos ocupacionais que levam ao óbito, constando dados de trabalhadores formais e informais, além de ser o principal sistema de informação da saúde para registro de doenças e agravos de notificação compulsória (BRASIL, 2018). 
Para a Vigilância em Saúde do Trabalhador (VISAT), o SINAN é um sistema de suma importância, propiciando a utilização dos dados para a análise da situação dos agravos ocorridos com trabalhadores, o que viabiliza a elaboração de medidas de intervenção e prevenção de acidentes graves (DRUMMOND AS, et al., 2018).

Se faz relevante então, conhecer o perfil produtivo do trabalhador e as atividades econômicas desenvolvidas nas regiões do estado, pois assim é possível apontar riscos aos quais os trabalhadores se expõem, para que se possa compreender a relação entre a atividade produtiva e os agravos sofridos pelo mesmo. Dessa forma, este estudo tem por objetivo descrever o perfil epidemiológico dos acidentes de trabalho graves notificados no estado da Bahia, no período de 2007 a 2017.

\section{MÉTODOS}

Trata-se de um estudo ecológico, descritivo, acerca dos ATG que ocorreram em trabalhadores, residentes no estado da Bahia, no período de 2007 a 2017. Para tanto, foi utilizado como fonte de dados o SINAN, obtidos através da Superintendência de Vigilância e Proteção da Saúde (SUVISA), da Secretaria da Saúde do Estado da Bahia (SESAB).

Com o fim de caracterizar a população, foram selecionadas variáveis em conformidade com a ficha de notificação/investigação de Acidente de Trabalho Grave do SINAN, sendo elas: características sociodemográficas (sexo, idade, raça/cor da pele e escolaridade); características do trabalho (ocupação, situação no mercado de trabalho, preenchimento da Classificação Nacional de Atividades Econômicas (CNAE), seção de atividade econômica e empresa e características do acidente (tipo de acidente, local do acidente, outros trabalhadores atingidos, emissão de Comunicação de Acidente de Trabalho (CAT), atendimento médico, regime de tratamento e evolução do caso.

A variável ocupação, é qualificada conforme a Classificação Brasileira de Ocupações (CBO), que organiza e descreve as ocupações do mercado brasileiro por categorias de famílias, na qual cada uma constitui um conjunto de ocupações análogas correspondendo a um domínio de trabalho mais amplo que aquele da ocupação, sendo o nível 1 a classificação mais generalizada e adotada para essa pesquisa (BRASIL, 2018).

Como se trata de um estudo com agregados de população, a unidade de observação dos dados foi apresentada de acordo com as nove macrorregiões de saúde ou Núcleos Regionais de Saúde (NRS) do estado da Bahia. As macrorregiões de saúde foram definidas conforme o Plano Diretor de Regionalização atual do estado e são constituídas por municípios, tendo uma ou mais cidades como centro polarizador de serviços de saúde de alta complexidade (BAHIA, 2018).

A análise dos dados foi baseada na estatística descritiva, obtendo-se as frequências absolutas e relativas e a incidência anual dos ATG (número de casos absolutos de ATG pela População em Idade Ativa (PIA) referente a cada ano pesquisado). A análise foi realizada através do programa Microsoft Excel Office ( ).

\section{RESULTADOS}

Foram notificados entre os anos 2007 e 2017, 20.345 (ATG), sendo a macrorregião Leste a que mais notificou (32,5\%), seguidas das macrorregiões Sul (21,3\%), Sudoeste $(10,8 \%)$ e Extremo Sul $(10,6 \%)$, respectivamente (Tabela 1).

No que tange ao período analisado, o coeficiente de incidência de ATG para cada 10.000 habitantes em PIA revelou aumento de 0,31 no ano de 2007 para 3,21 em 2017, um incremento de 93\%. Observa-se, entre os anos de 2015 e 2016, que houve uma discreta redução na incidência, apresentando uma elevação dos casos no ano em sequência (Figura 1).

Observou-se maior incidência dos ATG no período analisado, nas macrorregiões Extremo Sul $(3,32)$, Oeste $(2,12)$, Sudoeste $(1,51)$ e Leste $(1,46)$, sendo importante ressaltar um aumento expressivo da incidência para todos os núcleos regionais no ano de 2017 (Tabela 2). 
Tabela 1- Notificações de Acidentes de Trabalho Graves no estado da Bahia, segundo macrorregiões de saúde, entre os anos 2007 a 2017.

\begin{tabular}{ccc}
\hline Macrorregião & $\mathbf{N}$ & $\%$ \\
\hline Centro-Leste & 1755 & 8,7 \\
Centro-Norte & 470 & 2,4 \\
Extremo Sul & 2169 & 10,6 \\
Leste & 6615 & 32,5 \\
Nordeste & 791 & 3,9 \\
Norte & 358 & 1,8 \\
Oeste & 1635 & 8,0 \\
Sudoeste & 2207 & 10,8 \\
Sul & 4345 & 21,3 \\
\hline Total & $\mathbf{2 0 . 3 4 5}$ & $\mathbf{1 0 0}$
\end{tabular}

Fonte: Calazans MIP e Nery AA, 2020. Dados de: Diretoria de Vigilância e

Atenção à Saúde do Trabalhador (Divast) - Secretaria de Saúde do Estado da Bahia (Sesab), 2019.

Figura 1 - Série temporal dos coeficientes de incidência dos Acidentes de Trabalho Graves no estado da Bahia, no período de 2007 e 2017.

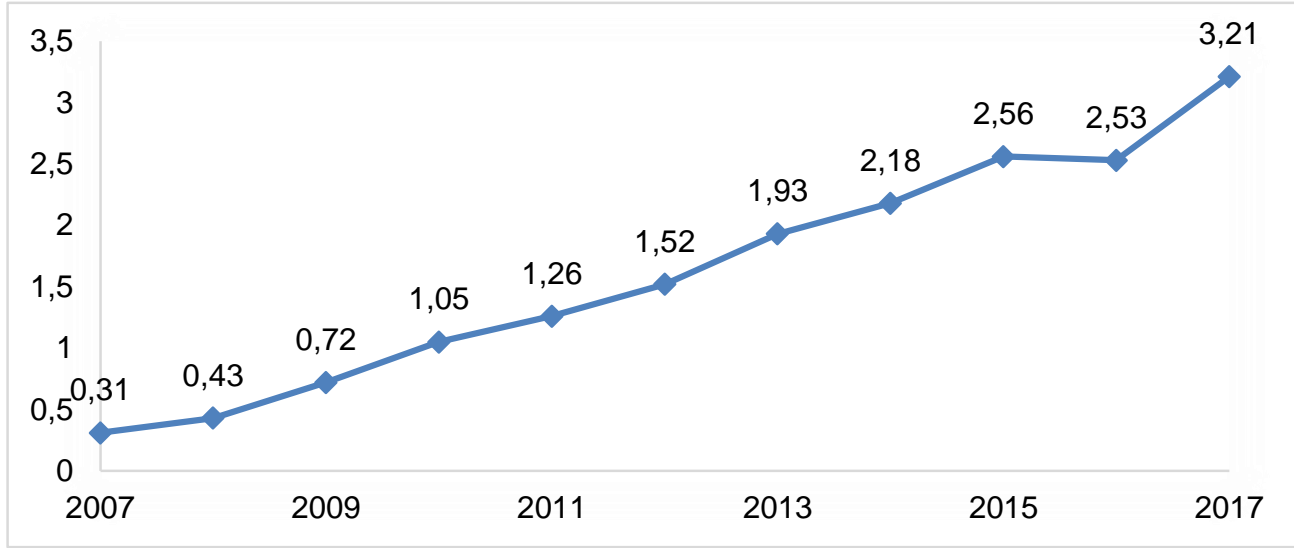

Fonte: Calazans MIP e Nery AA, 2020. Dados de: Diretoria de Vigilância e Atenção à Saúde do Trabalhador (Divast) - Secretaria de Saúde do Estado da Bahia (Sesab), 2019.

Tabela 2 - Coeficiente de incidência dos Acidentes de Trabalho Graves por macrorregiões de saúde, entre os anos 2007 a 2017.

\begin{tabular}{lcccccccccccc}
\hline Macrorregião & $\mathbf{2 0 0 7}$ & $\mathbf{2 0 0 8}$ & $\mathbf{2 0 0 9}$ & $\mathbf{2 0 1 0}$ & $\mathbf{2 0 1 1}$ & $\mathbf{2 0 1 2}$ & $\mathbf{2 0 1 3}$ & $\mathbf{2 0 1 4}$ & $\mathbf{2 0 1 5}$ & $\mathbf{2 0 1 6}$ & $\mathbf{2 0 1 7}$ & Total \\
\hline Centro-Leste & 0,13 & 0,17 & 0,24 & 0,7 & 0,67 & 0,99 & 1,53 & 1,54 & 1,99 & 2,63 & 2,68 & 1,24 \\
Centro-Norte & - & 0,02 & 0,07 & 0,17 & 0,71 & 1,2 & 1,05 & 1,11 & 1,15 & 2,01 & 2,6 & 0,95 \\
Extremo Sul & 1,97 & 2,6 & 2,21 & 4,78 & 3,9 & 2,65 & 3,81 & 3,59 & 5,15 & 2,36 & 3,19 & 3,32 \\
Leste & 0,08 & 0,23 & 0,34 & 0,2 & 0,46 & 0,57 & 1,83 & 1,63 & 2,99 & 2,99 & 4,14 & 1,46 \\
Nordeste & - & - & 0,07 & 0,2 & 0,78 & 1,37 & 2,46 & 3,55 & 2,18 & 1,41 & 0,99 & 1,22 \\
Norte & 0,04 & 0,03 & 0,04 & 0,41 & 0,32 & 0,71 & 0,37 & 0,92 & 0,61 & 0,45 & 0,73 & 0,43 \\
Oeste & 1,67 & 1,49 & 2,37 & 1,92 & 2,91 & 1,55 & 1,42 & 1,92 & 2,87 & 2,79 & 2,23 & 2,12 \\
Sudoeste & 0,13 & 0,08 & 0,23 & 0,74 & 1,48 & 3,19 & 2,21 & 2,1 & 1,96 & 2,06 & 2,25 & 1,51 \\
Sul & 0,39 & 0,87 & 2,49 & 3,24 & 3,04 & 3,29 & 2,98 & 4,81 & 3,37 & 3,66 & 5,81 & 3,1 \\
\hline Total & $\mathbf{0 , 3 1}$ & $\mathbf{0 , 4 3}$ & $\mathbf{0 , 7 2}$ & $\mathbf{1 , 0 5}$ & $\mathbf{1 , 2 6}$ & $\mathbf{1 , 5 2}$ & $\mathbf{1 , 9 3}$ & $\mathbf{2 , 1 8}$ & $\mathbf{2 , 5 6}$ & $\mathbf{2 , 5 3}$ & $\mathbf{3 , 2 1}$ & $\mathbf{1 , 6 4}$ \\
\hline
\end{tabular}

Fonte: Calazans MIP e Nery AA, 2020. Dados de: Diretoria de Vigilância e Atenção à Saúde do Trabalhador (Divast) - Secretaria de Saúde do Estado da Bahia (Sesab), 2019. 
Dentre as características sociodemográficas, os homens representam $87,8 \%$ dos casos notificados em todas as macrorregiões. A escolaridade foi uma variável que apresentou inconsistência nos dados, com $40,6 \%$ das notificações sem o registro desta informação. Observou-se que $25,5 \%$ destes possuíam ensino fundamental incompleto, no entanto, a macrorregião Leste, que apresentou mais notificações de ATG, teve o menor percentual de trabalhadores com ensino fundamental incompleto (Tabela 3).

Quanto à variável raça/cor da pele, percebe-se que $51,0 \%$ destes eram pardos. Estratificando por macrorregião, a Leste possuiu mais dados ignorados/não registrados (49,6\%). A faixa etária dos 20 aos 39 anos apresenta maior percentual $(56,6 \%)$ para as notificações de ATG em todas as macrorregiões.

Foram caracterizadas as variáveis do trabalho, dentre elas a variável que identifica CNAE não foi informada em $90,1 \%$ dos casos, o que prejudicou a identificação das atividades econômicas exercidas nas regiões. Em relação à ocupação, observou-se 37,4\% de trabalhadores inseridos na categoria de produção de bens e serviços I, sendo a macrorregião Leste a que mais apresentou trabalhadores nessa categoria $(45,7 \%)$ (Tabela 4).

Concernente à situação no mercado de trabalho, 32\% possuíam vínculo formal, sendo que a macrorregião Nordeste é a que apresenta mais trabalhadores nessa condição $(65,4 \%)$. Em relação aos empregados não registrados e autônomos, esses representavam 12,1\% e 23,3\%, respectivamente, com $19,2 \%$ de dados ignorados/não registrados. Quanto à variável empresa terceirizada, $40,5 \%$ das empresas contratantes não terceirizavam seus serviços e $46,9 \%$ dos dados foram ignorados/não registrados no total das macrorregiões.

Foram descritas as variáveis referentes aos acidentes. Quanto ao tipo de acidente $69,6 \%$ foram classificados como típicos, sendo a macrorregião Leste a mais notificante (81,3\%). As instalações do contratante foram apontadas como local do acidente mais frequente $(38,9 \%)$, com destaque para a macrorregião Nordeste $(61,4 \%)$. Os que ocorreram em via pública representaram $23,8 \%$. Em $75,3 \%$ dos casos não houve envolvimento de outros trabalhadores (Tabela 5).

A Comunicação de Acidente de Trabalho (CAT) não foi preenchida em 37,8\% das notificações em todas as macrorregiões, sendo pertinente destacar que dados ignorados/não registrados são da ordem de 40,5\%.

Em $91 \%$ das notificações houve necessidade de atendimento médico, sendo que $62 \%$ do tratamento dispensado ocorreram no ambiente hospitalar, com destaque para as macrorregiões Leste $(82,2 \%)$ e Oeste (68\%).

No que se refere à evolução dos casos, evidenciou-se que $37 \%$ dos trabalhadores obtiveram desfecho de incapacidade temporária e $20 \%$ cura. As macrorregiões Centro-leste (50,0\%), Extremo Sul (66,1\%) e Sudoeste $(51,5 \%)$ foram as que mais notificaram trabalhadores que, após o acidente, evoluíram para incapacidade temporária e a macrorregião Leste (36\%) para incapacidade permanente. Os óbitos pelo ATG corresponderam a $3,7 \%$ dos casos em todo o estado. 


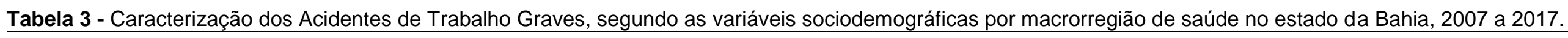

\begin{tabular}{|c|c|c|c|c|c|c|c|c|c|c|c|c|c|c|c|c|c|c|c|c|}
\hline \multirow{2}{*}{$\begin{array}{l}\text { Macrorregião de } \\
\text { Saúde }\end{array}$} & \multicolumn{2}{|c|}{ Centro-Leste } & \multicolumn{2}{|c|}{ Centro-Norte } & \multicolumn{2}{|c|}{ Extremo Sul } & \multicolumn{2}{|c|}{ Leste } & \multicolumn{2}{|c|}{ Nordeste } & \multicolumn{2}{|c|}{ Norte } & \multicolumn{2}{|c|}{ Oeste } & \multicolumn{2}{|c|}{ Sudoeste } & \multicolumn{2}{|c|}{ Sul } & \multicolumn{2}{|c|}{ Total } \\
\hline & 1755 & (\%) & 470 & (\%) & 2169 & $(\%)$ & 6615 & (\%) & 791 & $(\%)$ & 358 & (\%) & 1635 & $(\%)$ & 2207 & (\%) & 4345 & $(\%)$ & 20345 & $(\%)$ \\
\hline \multicolumn{21}{|l|}{ Sexo } \\
\hline Masculino & 1545 & 88 & 417 & 88,7 & 1975 & 91 & 5689 & 86 & 698 & 88,2 & 316 & 88,2 & 1478 & 90,4 & 1923 & 87,1 & 3830 & 88,1 & 17871 & 87,8 \\
\hline Feminino & 210 & 11,9 & 53 & 11,2 & 194 & 8,9 & 925 & 13,9 & 93 & 11,76 & 42 & 11,7 & 157 & 9,6 & 284 & 12,8 & 515 & 1,8 & 2473 & 12,1 \\
\hline Ignorado & - & & - & & - & & 1 & & - & & - & & - & & - & & - & & 1 & 0 \\
\hline \multicolumn{21}{|l|}{ Escolaridade } \\
\hline Analfabeto & 30 & 1,7 & 17 & 3,6 & 123 & 5,6 & 110 & 1,6 & 7 & 0,8 & 12 & 3,3 & 39 & 2,3 & 78 & 3,5 & 103 & 2,3 & 519 & 2,5 \\
\hline EF incompleto & 321 & 18,2 & 160 & 34 & 763 & 35,1 & 1092 & 16,5 & 142 & 17,9 & 96 & 26,8 & 585 & 35,7 & 756 & 34,2 & 1287 & 29,6 & 5202 & 25,5 \\
\hline EF completo & 60 & 3,4 & 25 & 5,3 & 123 & 5,6 & 261 & 3,9 & 29 & 3,6 & 12 & 3,3 & 106 & 6,4 & 129 & 5,8 & 638 & 14,6 & 1383 & 6,8 \\
\hline EM incompleto & 69 & 3,9 & 35 & 7,4 & 200 & 9,2 & 454 & 6,8 & 64 & 8 & 18 & 5 & 135 & 8,2 & 179 & 8,1 & 298 & 6,8 & 1452 & 7,1 \\
\hline EM completo & 179 & 10,2 & 73 & 15,5 & 440 & 20,2 & 736 & 11,1 & 113 & 14,2 & 47 & 13,1 & 251 & 15,3 & 292 & 13,2 & 542 & 12,4 & 2673 & 13,1 \\
\hline Ensino Superior & 39 & 2,2 & 14 & 2,9 & 75 & 3,4 & 141 & 2,1 & 27 & 3,4 & 10 & 2,7 & 40 & 2,4 & 71 & 3,2 & 142 & 3,2 & 559 & 2,7 \\
\hline Ignorado & 1032 & 58,8 & 137 & 29,1 & 430 & 19,8 & 3754 & 56,7 & 390 & 49,3 & 153 & 42,7 & 462 & 28,2 & 641 & 29 & 1275 & 29,3 & 8274 & 40,6 \\
\hline Não se aplica & 25 & 1,4 & 9 & 1,9 & 15 & 0,6 & 67 & 1 & 19 & 2,4 & 10 & 2,7 & 17 & 1 & 61 & 2,7 & 60 & 1,3 & 283 & 1,3 \\
\hline \multicolumn{21}{|l|}{ Raça/Cor } \\
\hline Amarela & 16 & 0,9 & 6 & 1,2 & 19 & 0,8 & 30 & 0,4 & 9 & 1,1 & - & - & 16 & 0,9 & 15 & 0,6 & 27 & 0,6 & 138 & 0,6 \\
\hline Branca & 121 & 6,8 & 60 & 12,7 & 346 & 15,9 & 735 & 11,1 & 78 & 9,8 & 37 & 10,3 & 244 & 14,9 & 366 & 16,5 & 288 & 6,6 & 2275 & 11,1 \\
\hline Preta & 153 & 8,7 & 43 & 9,1 & 304 & 14 & 829 & 12,5 & 161 & 20,3 & 32 & 8,9 & 130 & 7,9 & 205 & 9,2 & 674 & 15,5 & 2531 & 12,4 \\
\hline Parda & 1191 & 67,8 & 329 & 70 & 1377 & 63,4 & 1727 & 26,1 & 381 & 48,1 & 256 & 71,5 & 1096 & 67 & 1322 & 59,9 & 2696 & 62 & 10375 & 51 \\
\hline Indígena & - & - & 2 & 0,4 & 6 & 0,2 & 11 & 0,1 & 7 & 0,8 & 1 & 0,2 & 6 & 0,3 & 10 & 0,4 & 16 & 0,3 & 59 & 0,2 \\
\hline Ignorada & 274 & 15,6 & 30 & 6,3 & 117 & 5,3 & 3283 & 49,6 & 155 & 19,6 & 32 & 8,9 & 143 & 8,7 & 289 & 13 & 644 & 14,8 & 4967 & 24,4 \\
\hline \multicolumn{21}{|l|}{ Faixa Etária } \\
\hline$<10$ anos & 26 & 1,4 & 11 & 2,3 & 15 & 0,6 & 67 & 1 & 19 & 2,4 & 10 & 2,7 & 17 & 1 & 61 & 2,7 & 59 & 1,3 & 285 & 1,4 \\
\hline 10 a 19 anos & 139 & 7,9 & 14 & 2,9 & 125 & 5,7 & 373 & 5,6 & 35 & 4,4 & 30 & 8,3 & 97 & 5,9 & 115 & 5,2 & 222 & 5,1 & 1150 & 5,6 \\
\hline 20 a 39 anos & 968 & 55,1 & 245 & 52,1 & 1225 & 56,4 & 3705 & 56 & 487 & 61,5 & 195 & 54,4 & 980 & 59,9 & 1235 & 55,9 & 2360 & 54,3 & 11400 & 56 \\
\hline 40 a 59 anos & 541 & 30,8 & 161 & 34,2 & 722 & 33,2 & 2201 & 33,2 & 229 & 28,9 & 107 & 29,8 & 482 & 29,4 & 692 & 31,3 & 1447 & 33,3 & 6582 & 32,3 \\
\hline 60 e mais & 81 & 4,6 & 39 & 8,3 & 82 & 3,7 & 269 & 4 & 21 & 2,6 & 16 & 4,4 & 59 & 3,6 & 104 & 4,7 & 257 & 5,9 & 928 & 4,5 \\
\hline Total & 1755 & 100 & 470 & 100 & 2169 & 100 & 6615 & 100 & 791 & 100 & 358 & 100 & 1635 & 100 & 2207 & 100 & 4345 & 1000 & 20345 & 100 \\
\hline
\end{tabular}

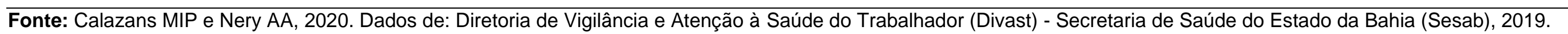


Tabela 4- Caracterização dos Acidentes de Trabalho Graves, segundo variáveis do trabalho por macrorregião de saúde no estado da Bahia, 2007 a 2017.

\begin{tabular}{|c|c|c|c|c|c|c|c|c|c|c|c|c|c|c|c|c|c|c|c|c|}
\hline \multirow{2}{*}{ Macrorregião de saúde } & \multicolumn{2}{|c|}{ Centro-Leste } & \multicolumn{2}{|c|}{ Centro-Norte } & \multicolumn{2}{|c|}{ Extremo Sul } & \multicolumn{2}{|c|}{ Leste } & \multicolumn{2}{|c|}{ Nordeste } & \multicolumn{2}{|c|}{ Norte } & \multicolumn{2}{|c|}{ Oeste } & \multicolumn{2}{|c|}{ Sudoeste } & \multicolumn{2}{|c|}{ Sul } & \multicolumn{2}{|c|}{ Total } \\
\hline & 1755 & $(\%)$ & 470 & $(\%)$ & 2169 & $(\%)$ & 6615 & $(\%)$ & 791 & $(\%)$ & 358 & $(\%)$ & 1635 & $(\%)$ & 2207 & $(\%)$ & 4345 & $(\%)$ & 20345 & $(\%)$ \\
\hline \multicolumn{21}{|l|}{ CNAE } \\
\hline Preenchida & 201 & 11,4 & 12 & 2,5 & 956 & 44 & 557 & 8,4 & 32 & 4 & 4 & 1,1 & 15 & 0,9 & 135 & 6,1 & 102 & 2,3 & 2014 & 9,8 \\
\hline Não preenchida & 1554 & 88,5 & 458 & 97,4 & 1213 & 55,9 & 6059 & 91,5 & 759 & 95,9 & 354 & 98,8 & 1620 & 99 & 2072 & 93,8 & 4243 & 97,6 & 18332 & 90,1 \\
\hline \multicolumn{21}{|l|}{ Ocupação } \\
\hline Forças Armadas, policiais e bombeiros & - & - & - & - & 10 & 0,4 & 24 & 0,3 & 3 & 0,3 & - & - & 5 & 0,3 & 4 & 0,1 & 16 & 0,3 & 62 & 0,3 \\
\hline Membros superiores do poder público & 14 & 0,7 & - & - & 19 & 0,8 & 53 & 0,8 & 7 & 0,8 & 2 & 0,5 & 37 & 2,2 & 20 & 0,9 & 65 & 1,4 & 217 & 1 \\
\hline Profissionais das ciências & 36 & 2 & 14 & 2,9 & 49 & 2,2 & 152 & 2,2 & 14 & 1,7 & 9 & 2,5 & 44 & 2,6 & 49 & 2,2 & 92 & 2,1 & 459 & 2,2 \\
\hline Técnicos de nível médio & 61 & 3,4 & 26 & 5,5 & 72 & 3,3 & 367 & 5,5 & 63 & 7,9 & 24 & 6,7 & 75 & 4,5 & 105 & 4,7 & 154 & 3,5 & 947 & 4,6 \\
\hline Serviços administrativos & 52 & 2,9 & 3 & 0,6 & 90 & 4,1 & 144 & 2,1 & 24 & 3 & 10 & 2,7 & 66 & 4 & 69 & 3,1 & 137 & 3,1 & 595 & 2,9 \\
\hline Vendedores do comércio & 125 & 7,1 & 30 & 6,3 & 330 & 15,2 & 1231 & 18,6 & 96 & 12,1 & 18 & 5 & 221 & 13,5 & 321 & 14,5 & 720 & 16,5 & 3092 & 15,1 \\
\hline Trabalhadores agropecuários & 228 & 12,9 & 199 & 42,3 & 630 & 29 & 713 & 10,7 & 122 & 15,4 & 119 & 33,2 & 429 & 26,2 & 458 & 20,7 & 1134 & 26 & 4032 & 19,8 \\
\hline $\begin{array}{l}\text { Trabalhadores da produção bens e } \\
\text { serviços I }\end{array}$ & 507 & 28,8 & 142 & 30,2 & 749 & 34,5 & 3026 & 45,7 & 340 & 42,9 & 118 & 32,9 & 557 & 34 & 873 & 39,5 & 1309 & 30,1 & 7621 & 37,4 \\
\hline $\begin{array}{l}\text { Trabalhadores da produção bens e } \\
\text { serviços II }\end{array}$ & 66 & 3,7 & 6 & 1,2 & 82 & 3,7 & 448 & 6,7 & 47 & 5,9 & 11 & 3 & 69 & 4,2 & 95 & 4,3 & 102 & 2,3 & 926 & 4,5 \\
\hline $\begin{array}{l}\text { Trabalhadores de manutenção e } \\
\text { reparação }\end{array}$ & 43 & 2,4 & 9 & 1,9 & 89 & 4,1 & 306 & 4,6 & 54 & 6,8 & 17 & 4,7 & 83 & 5 & 74 & 3,3 & 118 & 2,7 & 793 & 3,8 \\
\hline Estudante & 21 & 1,1 & 22 & 4,6 & 6 & 0,2 & 36 & 0,5 & 5 & 0,6 & 18 & 5 & 26 & 1,5 & 54 & 2,4 & 40 & 0,9 & 228 & 1,1 \\
\hline Dona de casa & 6 & 0,3 & 5 & 1 & 3 & 0,1 & 7 & 0,1 & 1 & 0,1 & 1 & 0,2 & 5 & 0,3 & 18 & 0,8 & 19 & 0,4 & 65 & 0,3 \\
\hline Aposentado/pensionista & 5 & 0,2 & 3 & 0,6 & 1 & 0 & 6 & 0 & 3 & 0,3 & - & - & 4 & 0,2 & 11 & 0,4 & 10 & 0,2 & 43 & 0,2 \\
\hline Desempregado Crônico & 8 & 0,4 & 1 & 0,2 & 2 & 0 & 23 & 0,3 & 2 & 0,2 & - & - & 7 & 0,4 & 3 & 0,1 & 97 & 2,2 & 143 & 0,7 \\
\hline Ignorado & 583 & 33,2 & 10 & 2,1 & 37 & 1,7 & 79 & 1,1 & 10 & 1,2 & 11 & 3 & 7 & 0,4 & 53 & 2,4 & 332 & 7,6 & 1124 & 5,5 \\
\hline \multicolumn{21}{|l|}{ Situação no mercado de trabalho } \\
\hline Empregado registrado & 393 & 22,3 & 86 & 18,2 & 1035 & 47,7 & 1785 & 26,9 & 518 & 65,4 & 91 & 25,4 & 751 & 45,9 & 695 & 31,4 & 1174 & 27 & 6528 & 32 \\
\hline Empregado não registrado & 206 & 11,7 & 61 & 12,9 & 320 & 14,7 & 453 & 6,8 & 77 & 9,7 & 79 & 22 & 246 & 15 & 315 & 14,2 & 710 & 16,3 & 2467 & 12,1 \\
\hline Autônomo & 315 & 17,9 & 158 & 33,6 & 456 & 21 & 1800 & 27,2 & 65 & 8,2 & 85 & 23,7 & 369 & 22,5 & 558 & 25,2 & 943 & 21,7 & 4749 & 23,3 \\
\hline Servidor público estatutário & 50 & 2,8 & 17 & 3,6 & 80 & 3,6 & 125 & 1,8 & 20 & 2,5 & 12 & 3,3 & 44 & 2,6 & 92 & 4,1 & 87 & 2 & 527 & 2,5 \\
\hline Servidor público celetista & 9 & 0,5 & 5 & 1 & 20 & 0,9 & 19 & 0,2 & 5 & 0,6 & 2 & 0,5 & 14 & 0,8 & 27 & 1,2 & 82 & 1,8 & 183 & 0,8 \\
\hline \multicolumn{21}{|l|}{ Empresa Terceirizada } \\
\hline Sim & 59 & 3,3 & 31 & 6,5 & 83 & 3,8 & 288 & 4,3 & 111 & 14 & 24 & 6,7 & 99 & 6 & 66 & 2,9 & 108 & 2,4 & 869 & 4,2 \\
\hline Não & 587 & 33,4 & 191 & 40,6 & 1563 & 72 & 1573 & 23,7 & 305 & 38,5 & 118 & 32,9 & 965 & 59 & 606 & 27,4 & 2351 & 54,1 & 8259 & 40,5 \\
\hline Ignorado & 1029 & 58,6 & 166 & 35,3 & 371 & 17,1 & 3780 & 57,1 & 358 & 45,2 & 181 & 50,5 & 502 & 30,7 & 1465 & 66,3 & 1700 & 39,1 & 9552 & 46,9 \\
\hline Não se aplica & 80 & 4,5 & 82 & 17,4 & 152 & 7 & 974 & 14,7 & 17 & 2,1 & 35 & 9,7 & 69 & 4,2 & 70 & 3,1 & 186 & 4,2 & 1665 & 8,1 \\
\hline Total & 1755 & 100 & 470 & 100 & 2169 & 100 & 6615 & 100 & 791 & 100 & 358 & 100 & 1635 & 100 & 2207 & 100 & 4345 & 100 & 20345 & 100 \\
\hline
\end{tabular}

Fonte: Calazans MIP e Nery AA, 2020. Dados de: Diretoria de Vigilância e Atenção à Saúde do Trabalhador (Divast) - Secretaria de Saúde do Estado da Bahia (Sesab), 2019. 
Tabela 5 - Caracterização dos Acidentes de Trabalho Graves, segundo variáveis do acidente por macrorregião de saúde no estado da Bahia, 2007 a 2017.

\begin{tabular}{|c|c|c|c|c|c|c|c|c|c|c|c|c|c|c|c|c|c|c|c|c|}
\hline \multirow{2}{*}{ Macrorregião de saúde } & \multicolumn{2}{|c|}{ Centro-Leste } & \multicolumn{2}{|c|}{ Centro-Norte } & \multicolumn{2}{|c|}{ Extremo-Sul } & \multicolumn{2}{|c|}{ Leste } & \multicolumn{2}{|c|}{ Nordeste } & \multicolumn{2}{|c|}{ Norte } & \multicolumn{2}{|c|}{ Oeste } & \multicolumn{2}{|c|}{ Sudoeste } & \multicolumn{2}{|c|}{ Sul } & \multicolumn{2}{|c|}{ Total } \\
\hline & 1755 & $(\%)$ & 470 & $(\%)$ & 2169 & $(\%)$ & 6615 & $(\%)$ & 791 & $(\%)$ & 358 & $(\%)$ & 1635 & $(\%)$ & 2207 & $(\%)$ & 4345 & $(\%)$ & 20345 & $(\%)$ \\
\hline \multicolumn{21}{|l|}{ Tipo de acidente } \\
\hline Típico & 896 & 51 & 316 & 67,2 & 1661 & 76,5 & 5380 & 81,3 & 597 & 75,4 & 213 & 59,5 & 1041 & 63,6 & 1227 & 55,6 & 2848 & 65,5 & 14179 & 69,6 \\
\hline Trajeto & 210 & 11,9 & 79 & 16,8 & 439 & 20,2 & 852 & 12,8 & 111 & 14 & 51 & 14,2 & 374 & 22,8 & 616 & 27,9 & 1061 & 24,4 & 3793 & 18,6 \\
\hline Ignorado & 649 & 36,9 & 75 & 15,9 & 69 & 3,1 & 383 & 5,7 & 83 & 10,4 & 94 & 26,2 & 220 & 13,4 & 364 & 16,4 & 436 & 10 & 2373 & 11,6 \\
\hline \multicolumn{21}{|l|}{ Local do acidente } \\
\hline Instalações contratante & 551 & 31,4 & 147 & 31,2 & 1223 & 56,3 & 2169 & 32,7 & 486 & 61,4 & 153 & 42,7 & 793 & 48,5 & 697 & 31,5 & 1706 & 39,2 & 7925 & 38,9 \\
\hline Via pública & 293 & 16,7 & 102 & 21,7 & 635 & 29,2 & 1301 & 19,6 & 166 & 20,9 & 56 & 15,6 & 438 & 26,7 & 539 & 24,4 & 1312 & 30,2 & 4842 & 23,8 \\
\hline Instalações de terceiros & 148 & 8,4 & 62 & 13,1 & 147 & 6,7 & 789 & 11,9 & 74 & 9,3 & 51 & 14,2 & 152 & 9,3 & 214 & 9,7 & 383 & 8,8 & 2020 & 9,9 \\
\hline Domicílio próprio & 114 & 6,5 & 81 & 17,2 & 69 & 3,1 & 399 & 6 & 16 & 2 & 49 & 13,6 & 140 & 8,5 & 259 & 11,7 & 381 & 8,7 & 1508 & 7,4 \\
\hline Ignorado & 649 & 36,9 & 78 & 16,6 & 95 & 4,3 & 1957 & 29,5 & 49 & 6,1 & 49 & 13,6 & 112 & 6,8 & 498 & 22,5 & 563 & 12,9 & 4050 & 19,9 \\
\hline \multicolumn{21}{|l|}{ Outros trab. atingidos } \\
\hline Sim & 112 & 6,3 & 37 & 7,8 & 195 & 8,9 & 418 & 6,3 & 88 & 11,1 & 35 & 9,7 & 187 & 11,4 & 196 & 8,8 & 386 & 8,8 & 1654 & 8,1 \\
\hline Não & 939 & 53,5 & 379 & 80,6 & 1912 & 88,1 & 4757 & 71,9 & 605 & 76,4 & 277 & 77,3 & 1329 & 81,2 & 1650 & 74,7 & 3474 & 79,9 & 15322 & 75,3 \\
\hline Ignorado & 704 & 40,1 & 54 & 11,4 & 62 & 2,8 & 1440 & 21,7 & 98 & 12,3 & 46 & 12,8 & 119 & 7,2 & 361 & 16,3 & 485 & 11,1 & 3369 & 16,5 \\
\hline \multicolumn{21}{|l|}{ CAT } \\
\hline Sim & 226 & 12,8 & 63 & 13,4 & 270 & 12,4 & 1105 & 16.7 & 115 & 14.5 & 56 & 15,6 & 215 & 13,1 & 111 & 5 & 452 & 10,4 & 2613 & 12.8 \\
\hline Não & 508 & 28,9 & 257 & 54,6 & 1031 & 47,5 & 1413 & 21,3 & 271 & 34,2 & 135 & 37,7 & 698 & 42,6 & 719 & 32,5 & 2669 & 61,4 & 7701 & 37,8 \\
\hline Não se aplica & 131 & 7,4 & 47 & 10 & 137 & 6,3 & 1190 & 17,9 & 20 & 2,5 & 20 & 5,5 & 55 & 3,3 & 44 & 1,9 & 136 & 3,1 & 1780 & 8,7 \\
\hline Ignorado & 890 & 50,7 & 103 & 21,9 & 731 & 33,7 & 2907 & 43,9 & 385 & 48,6 & 147 & 41 & 667 & 40,8 & 1333 & 60,4 & 1088 & 25 & 8251 & 40,5 \\
\hline \multicolumn{21}{|l|}{ Atendimento médico } \\
\hline Sim & 1555 & 88,6 & 433 & 92,1 & 2038 & 93,9 & 6140 & 92,8 & 663 & 83,8 & 304 & 84,9 & 1498 & 91,6 & 1885 & 85,4 & 4001 & 92 & 18517 & 91 \\
\hline Não & 62 & 3,5 & 13 & 2,7 & 100 & 4,6 & 131 & 1,9 & 63 & 7,9 & 19 & 5,3 & 62 & 3,7 & 107 & 4,8 & 223 & 5,1 & 780 & 69,6 \\
\hline Ignorado & 138 & 7,8 & 24 & 5,1 & 31 & 1,4 & 344 & 5,2 & 65 & 8,2 & 35 & 9,7 & 75 & 4,5 & 215 & 9,7 & 121 & 2,7 & 1048 & 5,1 \\
\hline \multicolumn{21}{|l|}{ Regime de tratamento } \\
\hline Hospitalar & 1021 & 58,1 & 229 & 48,7 & 838 & 38,6 & 5438 & 82,2 & 364 & 46 & 157 & 43,8 & 1113 & 68 & 1243 & 56,3 & 2221 & 51,1 & 12624 & 62 \\
\hline Ambulatorial & 394 & 22,4 & 151 & 32,1 & 262 & 12 & 657 & 9,9 & 264 & 33,3 & 113 & 31,5 & 295 & 18 & 459 & 20,8 & 1456 & 33,5 & 4051 & 19,9 \\
\hline Ambos & 127 & 7,2 & 35 & 7,4 & 934 & 43 & 98 & 1,4 & 15 & 1.9 & 26 & 7,2 & 42 & 2.5 & 74 & 3,3 & 255 & 5,8 & 1606 & 7.8 \\
\hline Ignorado & 213 & 12,1 & 55 & 11,7 & 135 & 6,2 & 422 & 6,3 & 148 & 18,7 & 62 & 17,3 & 185 & 11,3 & 431 & 19,5 & 413 & 9,5 & 2064 & 10,1 \\
\hline \multicolumn{21}{|l|}{ Evolução do caso } \\
\hline Cura & 219 & 12,4 & 121 & 25,7 & 465 & 21,4 & 842 & 12,7 & 232 & 29,3 & 137 & 38,2 & 426 & 26 & 496 & 22,4 & 1147 & 26,4 & 4085 & 20 \\
\hline Inc. temporária & 879 & 50 & 208 & 44,2 & 1434 & 66,1 & 1071 & 16,1 & 232 & 29,3 & 75 & 20,9 & 789 & 48,2 & 618 & 28 & 2240 & 51,5 & 7546 & 37 \\
\hline Inc. parcial permanente & 130 & 7,4 & 56 & 11,9 & 77 & 3,5 & 2384 & 36 & 35 & 4,4 & 19 & 5,3 & 135 & 8,2 & 89 & 4 & 262 & 6 & 3187 & 15,8 \\
\hline Inc. total permanente & 27 & 1,5 & 20 & 4,2 & 9 & 0,4 & 31 & 0,4 & 8 & 1 & 6 & 1,6 & 32 & 1,9 & 13 & 0,5 & 33 & 0,7 & 179 & 0,9 \\
\hline Óbito pelo acidente & 43 & 2.4 & 12 & 2,5 & 102 & 4.7 & 124 & 1.8 & 56 & 7 & 26 & 7,2 & 91 & 5,5 & 106 & 4.8 & 209 & 4,8 & 769 & 3,8 \\
\hline Obito por outras causas & 16 & 0,9 & - & - & 10 & 0,4 & 4 & 0 & 3 & 0,3 & 1 & 0,2 & - & - & 3 & 0,1 & 9 & 0,2 & 46 & 0,2 \\
\hline Outra & 30 & 1,7 & 7 & 1,4 & 12 & 0,5 & 51 & 0,7 & 7 & 0,8 & 12 & 3,3 & 33 & 2 & 22 & 0,9 & 22 & 0,5 & 196 & 0,9 \\
\hline Ignorado & 411 & 23,4 & 46 & 9,7 & 60 & 2,7 & 2108 & 31,8 & 218 & 27,5 & 82 & 22,9 & 129 & 7,8 & 860 & 38.9 & 423 & 9,7 & 4337 & 21,3 \\
\hline Total & 1755 & 100 & 470 & 100 & 2169 & 100 & 6615 & 100 & 791 & 100 & 358 & 100 & 1635 & 100 & 2207 & 100 & 4345 & 100 & 20345 & 100 \\
\hline
\end{tabular}

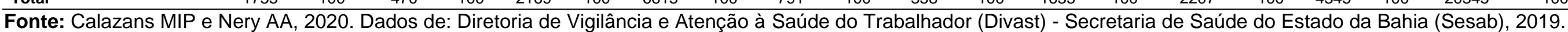




\section{DISCUSSÃO}

Nas últimas décadas, a Bahia tem atraído grandes empreendimentos, implantação de indústrias das mais diversas atividades econômicas e produtivas, com mão de obra crescente e aumento da empregabilidade em todas as regiões do estado, fatos que podem contribuir para a ocorrência de eventos em saúde relacionados ao trabalho, como os acidentes (CNI, 2017; NOBRE LCC, 2010).

Alguns estudos que analisaram ATG, partindo de dados secundários, evidenciaram um aumento das notificações em diversos estados brasileiros, corroborando com o elevado número de casos de acidentes graves no estado da Bahia no período analisado. Esse crescimento pode estar relacionado às grandes mudanças ocorridas nas organizações e nos processos de trabalho, pela chamada reestruturação produtiva, mas também devido à melhoria e busca pela qualidade do processo de notificação (SCUSSIATO LA, et al., 2013; CARDOSO EM, 2014; CARDOSO MG, 2016; BAHIA, 2016; NOVAIS DG e RIBEIRO LAO, 2015; SCUSSIATO LA, et al., 2010; SOUTO CC, et al., 2016).

Devido às diferenças regionais existentes no estado referentes às atividades econômicas e produtivas específicas, justifica-se a observação dos casos de ATG por Núcleos Regionais ou macrorregião de saúde. Destas, a macrorregião Leste, foi identificada como a mais notificante, cuja sede é Salvador, capital do estado, pois concentra o maior complexo industrial do Hemisfério Sul, o Polo Industrial de Camaçari, que constitui mais de 90 empresas dos setores químico e petroquímico, sendo referência no setor automotivo, bem como de outros campos de produção de bens e serviços do setor primário e terciário (CNI, 2017). Compreende-se, desta forma, as maiores notificações para essa região, tendo em vista o perfil produtivo do trabalhador e os riscos inerentes a ocupações dessa natureza (FILHO APG e RAMOS MF, 2010).

Observou-se um aumento expressivo na incidência dos acidentes na última década em todo o estado, especialmente para algumas macrorregiões, sendo evidenciado tal aumento também por outros estudos (CAVALCANTE CAA, et al., 2015; SCUSSIATO LA, et al., 2013; CARDOSO EM, 2014). Contudo, estes dados não representam a real dimensão dos acidentes graves no país e mais especificamente no estado da Bahia, tendo em vista as limitações existentes, como o não preenchimento das CAT, a subnotificação das fichas de notificação/investigação e registro no SINAN.

A literatura acerca do tema é enfática em relação à predominância dos acidentes graves nos homens, sendo este gênero majoritariamente ocupante de atividades laborativas que representam maior risco para acidentes (CARDOSO MG, 2016; SOUTO CC, et al., 2016; BALDO RCS, SPAGNUOLO RS e ALMEIDA IM, 2015; NOGUEIRA JM e SILVA SM, 2018).

A categoria de faixa etária na qual se mostrou mais ocorrente (20-39) anos, é a que representa a alta produtividade no mercado de trabalho, levando a prejuízos na economia do estado e implicações sociais aos trabalhadores vitimados, tendo em vista as consequências que os acidentes graves trazem.

Desta maneira, é possível entender quando se observa as características sexo e faixa etária, que os acidentes graves nesses trabalhadores podem estar relacionados às ideias do ser homem na sociedade, bem como aos fatores de risco mais prevalentes neste segmento populacional, como: o excesso de velocidade no trânsito, o uso de álcool e/ou outras drogas, o sono/fadiga, o não uso do cinto de segurança e de Equipamentos de Proteção Individual (EPI) e, da não observância e inadequação das normas de segurança no ambiente de trabalho (SOUTO CC, et al., 2016; FILHO APG e RAMOS MF, 2010; CAMPOS AG e GURGEL AM, 2016).

Foi identificada grande subnotificação/subregistro para as variáveis escolaridade e raça/cor da pele. Dos dados notificados, observou-se que os trabalhadores eram maioria de cor parda e que possuíam até o momento do acidente grave ensino fundamental incompleto, o que vale destacar que a baixa escolaridade pode ser justificada pelo fato de haver maiores ofertas de empregos com baixa exigência em escolaridade para ocupações, como a construção civil e a produção de bens e serviços (agricultura, manutenção e reparo e serviços no comércio) (CARDOSO MG, et al., 2016). 
Sobre as variáveis que caracterizam o trabalho, em mais de $90 \%$ dos casos a variável CNAE não foi preenchida, o que dificulta o conhecimento das atividades econômicas que mais ocasionam acidentes nas macrorregiões. Alguns estudos também trazem essa constatação, o que permite inferir que os profissionais responsáveis pela notificação e investigação dos agravos em saúde podem apresentar dificuldades na compreensão de conceitos e utilização da classificação pela CNAE, o que interfere sobremaneira no diagnóstico situacional de saúde dos trabalhadores e ainda no planejamento de ações voltadas à saúde e segurança destes (DRUMMOND AS, et al., 2018; ALVARES JK, et al., 2015).

Suscita-se, desta forma, a necessidade da padronização dos campos de preenchimento obrigatório desta e de outras variáveis, bem como o conhecimento acerca das classificações e códigos para as classes e famílias ocupacionais, como da lista da CBO. Sobre este aspecto, Galdino A, Santana VS e Ferrite S (2017) enfatizam a importância da melhoria da qualidade do registro nos sistemas de informação em saúde, que deve ser impulsionada mediante a estruturação e preenchimento de campos obrigatórios das fichas.

Acerca da variável ocupação, foi observado que boa parte dos trabalhadores pertencia à categoria de 'trabalhadores da produção de bens e serviços l', que, segundo a CBO compreendem as ocupações com atividades que não exigem conhecimento especializado. São os trabalhadores da produção extrativa, da construção civil e da indústria de transformação (processos relativos à forma e montagem de produtos) (BRASIL, 2018).

Conforme apontam Nascimento FC e Salim CA (2018), trabalhadores da construção civil ainda estão entre os que mais se acidentam, embora tenha havido um declínio dos acidentes nessa categoria quando comparado à década de 1990. Cavalcante CAA, et al. (2015) e Miranda FMD, et al. (2012) afirmam que não somente os trabalhadores da construção civil, como também dos transportes, pois são atividades que, além de muito arriscadas, são as que mais absorvem mão de obra temporária e em alta rotatividade.

Quanto à situação no mercado de trabalho, ficou evidenciado que os trabalhadores formais foram mais vitimados pelos ATG. Os casos ignorados/não preenchidos destacam-se e se faz pertinente frisar a importância da completude dessas informações para ter conhecimento da dimensão do vínculo empregatício daqueles que sofrem os acidentes, tendo em vista o fato de serem os trabalhadores de contratações temporárias, informais e autônomos, os mais suscetíveis aos riscos de acidentes graves, justificados, segundo Druck G (2011), pelas várias formas que a precarização se apresenta para o trabalho e o trabalhador, como ausência de proteção social e submissão à intensificação do trabalho em condições precárias em prejuízo da sua saúde e perda da identidade individual e coletiva.

A variável empresa terceirizada apresentou importante subnotificação, especificamente nas macrorregiões Centro-Leste e Leste, nas quais há intensificação das atividades produtivas relativas às indústrias dos ramos petroquímico, de transformação (mais notadamente o setor automobilístico), bem como de produção de diversos serviços do setor terciário. Importante destacar que as ocupações para esses setores, são, em grande parte, devido às terceirizações de serviços.

Marcado como resultado da reestruturação produtiva, desde a década de 1990 no Brasil, o modelo de terceirização pós-fordista é empregado em grandes empresas tanto nacionais quanto multinacionais, nos quais serviços com as chamadas atividades-meio são distribuídas a empresas menores e com serviço especializado (FIGUEIREDO M, et al., 2007).

Nesse modelo de flexibilização do trabalho, Druck G e Franco T (2006), afirmam que há transferência de responsabilidades, tanto de gestão, quanto dos custos com a mão de obra a um terceiro, tirando a obrigação da empresa com os gastos trabalhistas.

Muitos casos de acidentes graves com trabalhadores terceirizados não são apropriadamente notificados, sendo registrados como doença, ou registrados nas CAT com atrasos, mesmo havendo a recomendação para que, qualquer pessoa, entidade, empresa ou instituição, tendo conhecimento de um ou mais casos graves de acidente de trabalho, comunique às unidades de saúdes e de especialização em Saúde do Trabalhador, como os CEREST e as unidades sentinelas com serviços de média e alta complexidade. 
Deve-se ressaltar que, o não preenchimento da CAT é uma realidade presente para trabalhadores registrados com contratos efetivos, mas que tal fato pode ser ainda mais presente em trabalhadores com ocupações flexibilizadas. O presente estudo demonstrou o quanto esta variável é tratada com pouca importância, mesmo sendo um documento de registro limitado para uma parcela de trabalhadores e muitas vezes preenchido apenas pela intenção de cobrir o trabalhador celetista com o Seguro de Acidentes de Trabalho. A subnotificação compromete desta maneira, a qualidade das informações, a análise da situação de saúde do trabalhador e prejudica ações de planejamento e estratégia de enfrentamento para esse tipo de agravo (ALVARES JK, et al., 2015).

Em relação ao tipo de acidente, observou-se o predomínio de acidentes típicos em todas as macrorregiões do estado. Estudos afirmam que grande parte dos acidentes graves se constituem como típicos, especialmente aqueles que ocorrem em instalações do empregador (NOVAIS DG e RIBEIRO LAO, 2015; SCUSSIATO LA, et al., 2010). Desta forma, entende-se que a ocorrência desse tipo de acidente tem relação com a organização do trabalho e em como se dão os processos de trabalho, necessitando da adoção de medidas de prevenção, controle e ações através de diversos setores interinstitucionais governamentais e das instituições contratantes.

Destaca-se ainda que, embora nesse estudo os acidentes de trajeto não tenham sido tão ocorrentes no período analisado, há estudos realizados em outros estados que confirmam que nos últimos 25 anos acidentes dessa natureza tem aumentado exponencialmente em todo o Brasil, estando relacionados aos processos de trabalho, a partir do momento em que as mudanças ocasionadas pela reestruturação dos processos produtivos ampliaram os espaços de exercício profissional e os expandiram para as ruas, expondo o trabalhador a novos riscos e intensificando os já existentes (LOURENÇO EAS, 2011; CORDEIRO R , 2018).

Para as variáveis atendimento, regime de tratamento e evolução do caso, foram observadas boa cobertura dos dados. Em relação aos atendimentos, estes ocorreram em mais de $90 \%$, detectável em todas as macrorregiões de saúde, sendo o primeiro tratamento dispensado pela unidade hospitalar, entretanto, cabe salientar que o local de atendimento depende da gravidade do acidente e demanda e oferta dos serviços de saúde local/regional. Em outros estudos são descritos o serviço ambulatorial como o mais utilizado (DRUMOND AS, et al., 2018; CARDOSO MG, et al., 2016).

Referente à evolução do caso, a maior frequência foi para a incapacidade temporária. Contudo, a observação dos casos por macrorregião revela que as de maior destaque para esse desfecho são as macrorregiões Extremo Sul, Sul e Centro-leste. Alguns estudos também trazem informações semelhantes concernentes à frequência de casos para incapacidade temporária (CAVALCANTE CAA, et al., 2015; SCUSSIATO LA, et al., 2013). Chama-se atenção para a macrorregião Leste que, dos ATG notificados, resultaram mais em incapacidades parciais permanentes. Na localização dessa macrorregional de saúde (região metropolitana e capital do estado) estão concentradas uma alta produtividade com setores ocupacionais de alto risco para o trabalhador.

Embora tenha sido observado uma frequência menor para os óbitos, as macrorregiões Sudoeste, Sul e Extremo Sul, revelam dados preocupantes. Tais regiões possuem atividades produtivas mais voltadas ao setor primário (pecuária, agricultura) e de indústrias de transformação (minérios) e de bens de consumo (têxtil, alimentos), bem como o ramo da construção civil, bastante crescente na última década 15, o que leva ao entendimento de que deve haver nos seus respectivos núcleos regionais e redes de ações de saúde do trabalhador (CEREST e unidades sentinela) olhares voltados a esses tipos de ocupações e o risco que representam a esses trabalhadores.

Assim, mesmo diante das limitações apresentadas pelo SINAN, tal sistema se revela essencial para o conhecimento da situação de saúde e segurança dos trabalhadores, o que permitiu delinear o perfil produtivo e chegar a uma interpretação da situação de trabalho e de saúde do trabalhador baiano, no entendimento de que há uma conexão entre a reestruturação produtiva atuante no país nas últimas três décadas com o aumento dos acidentes graves no período analisado, mesmo que não se tenha buscado inferir a sua causalidade, por ter este estudo um propósito descritivo. 


\section{CONCLUSÃO}

Através deste estudo foi possível conhecer o perfil dos acidentes de trabalho graves no estado da Bahia em um determinado período, as caraterísticas sociodemográficas, ocupacionais e de ocorrência dos acidentes e dada a importância destes para a Saúde Pública, compreende-se que é necessária e prioritária as ações de prevenção e promoção da segurança nos ambientes laborais, desde que haja uma forte articulação interinstitucional e no âmbito social das três esferas de governo, empresa/instituição empregadora e o trabalhador. Aos gestores de saúde e demais profissionais é imprescindível que haja um maior envolvimento destes no planejamento de ações voltadas à melhoria das condições de trabalho e saúde, levando em consideração o perfil produtivo do trabalhador em cada macrorregião de saúde.

\section{REFERÊNCIAS}

1. ALVARES JK, et al. Avaliação da completitude das notificações compulsórias relacionadas ao trabalho registradas por município polo industrial no Brasil, 2007 - 2011. Revista Brasileira de Epidemiologia. 2015; 18(1): 123-136.

2. ANDRADE WS, SANTOS KOB. Internações hospitalares por acidentes relacionadas ao trabalho notificadas na Bahia. Revista Pesquisa em Fisioterapia. 2018; 8(2): 208-215.

3. BAHIA. Secretaria da Saúde do Estado. Superintendência de Vigilância e Proteção da Saúde. Centro Estadual de Referência em Saúde do Trabalhador. Manual de normas e rotinas do sistema de informação de agravos de notificação - SINAN. organizadores Norma Suely Souto Souza; Delsuc Evangelista Filho; Mônica Moura da Costa e Silva. Salvador: CESAT; 2009; 58 p.

4. BAHIA. Secretaria de Saúde do Estado. Portaria no 1.411 de 03 de novembro de 2016. Define a Lista Estadual de Notificação Compulsória de doenças, agravos e eventos de saúde pública nos serviços de saúde públicos e privados em todo o território estadual, nos termos do anexo, e dá outras providências. Diário Oficial do Estado da Bahia, Salvador, BA, no 22.052, 8 nov. 2016; p. 24-25.

5. BAHIA. Secretaria da Saúde do estado da Bahia [Internet]. Municípios e Regionalização. 2018.

6. BALDO RCS, SPAGNUOLO RS, ALMEIDA IM. O Serviço Integrado de Atendimento ao Trauma em Emergência (SIATE) como fonte de informações de acidentes de trabalho em Londrina, PR. Revista Brasileira de Saúde Ocupacional. 2015; 40(132): 147-155.

7. BRASIL. Ministério da Saúde. Portaria n. $777 / G M$, de 28 de abril de 2004. Dispõe sobre os procedimentos técnicos para a notificação compulsória de agravos à saúde do trabalhador em rede de serviços sentinela específica, no Sistema Único de Saúde - SUS. Diário Oficial da União, Poder Executivo, Brasília, DF, no 81, 29 abr. 2004; Seção 1, p. 37-38.

8. BRASIL. Ministério da Saúde [Internet]. Portaria n.ำ 204, de 17 de fevereiro de 2016. Define a Lista Nacional de Notificação Compulsória de doenças, agravos e eventos de saúde pública nos serviços de saúde públicos e privados em todo o território nacional, nos termos do anexo, e dá outras providências. Brasília-DF, 2016.

9. BRASIL. Ministério do Trabalho e Emprego [Internet]. Classificação Brasileira de Ocupações. 2018.

10. BRASIL. Ministério da Saúde [Internet]. Sistema de Informação de Agravos de Notificação - SINAN. 2018.

11. CAMPOS AG, GURGEL AM. Acidentes de trabalho graves e atividades produtivas nas regiões administrativas de saúde em Pernambuco: uma análise a partir da identificação de aglomerados produtivos locais. Revista Brasileira de Saúde Ocupacional. 2016; 41(15): 01-12.

12. CARDOSO EM. Morbimortalidade relacionada ao trabalho no estado do Amazonas, Brasil, $2000-2011$. Epidemiologia e Serviços de Saúde. 2014; 23(1): 143-153.

13. CARDOSO MG, et al. Caracterização das ocorrências de acidentes de trabalho graves. Arquivos Ciências da Saúde. 2016; 23(4): 83-88.

14. CAVALCANTE CAA, et al. Análise crítica dos acidentes de trabalho no Brasil. Revista de Atenção em Saúde. 2015; 13(44): 100-109.

15. CONFEDERAÇÃO NACIONAL DA INDÚSTRIA (CNI) [Internet]. Categoria de serviço - guia básico do estado da Bahia. 2017.

16. CORDEIRO R. A inadequação da classificação oficial dos acidentes de trabalho no Brasil. Cadernos de Saúde Pública, 2018; 34(2): e00173016, 2018.

17. DRUCK G. Trabalho, precarização e resistências: novos e velhos desafios? Caderno CRH. 2011; 24(1): 37-57, 2011.

18. DRUCK G, FRANCO T. Terceirização e precarização: o binômio antissocial em indústrias. Em: Druck G, Franco T, organizadores. A perda da razão social do trabalho: terceirização e precarização. 1' reimpressão. São Paulo: Boitempo; 2016; cap. 6, p. 97-118.

19. DRUMOND AS, et al. Acidentes de trabalho grave notificados no Sistema de Informação de Agravos de Notificação (Sinan) no município de Belo Horizonte no período de 2008 a 2014. 2018. [Internet] Revista Médica de Minas Gerais.

20. FIGUEIREDO M, et al. Reestruturação produtiva, terceirização e relações de trabalho na indústria petrolífera offshore da Bacia de Campos (RJ). Gestão \& Produção. 2007; 14(1): 55-68.

21. FILHO APG, RAMOS MF. Trabalho Decente e segurança do trabalhador: análise dos acidentes de trabalho na Bahia no período de 2005 a 2009. Bahia Análise e Dados. 2010; 20(2/3): 327-337.

22. GALDINO A, SANTANA VS, FERRITE S. Os Centros de Referência em Saúde do Trabalhador e a notificação de acidentes de trabalho no Brasil. Cadernos de Saúde Pública. 2012; 28(1): 145-159. 
23. LOURENÇO EAS. Agravos à saúde dos trabalhadores no Brasil: alguns nós críticos. PEGADA-A Revista da Geografia do Trabalho. 2011; 12(1): 03-33.

24. MIRANDA FMD, et al. Caracterização das vítimas e dos acidentes de trabalho fatais. Revista Gaúcha de Enfermagem. 2012;33(2):45-51.

25. NASCIMENTO FC, SALIM CA. Política de prevenção de acidentes na construção civil: uma análise das práticas da inspeção do trabalho. Revista Psicologia, Organizações e Trabalho. 2018; 18(1): 299-305.

26. NOBRE LCC. Relações entre produção, trabalho, ambiente e saúde: a contribuição do Sistema Único de Saúde para a conquista do Trabalho Decente. Bahia Análise e Dados. 2010; 20(2/3): 339-348.

27. NOGUEIRA JM, SILVA SM. Perfil dos acidentes de trabalho ocorridos na região leste da cidade de São Paulo. Arquivos Médicos dos Hospitais e da Faculdade de Ciências Médicas da Santa Casa de São Paulo. 2018; 62(2): 59-62.

28. NOVAIS DG, RIBEIRO LAO. Perfil epidemiológico dos acidentes de trabalho graves notificados do ano de 2011 a novembro de 2014 no município de Araguatins-TO, Brasil. Humanidades \& Inovação. 2015; 2(2): 6-1.

29. OBSERVATÓRIO DIGITAL DE SAÚDE E SEGURANÇA DO TRABALHO [Internet]. Ministério Público do Trabalho e Organização Internacional do Trabalho: Gastos atualizados da Previdência com Benefícios Acidentários. 2018.

30. SCUSSIATO LA, et al. Análise dos agravos relacionados ao trabalho notificados pela unidade saúde do trabalhador. Revista Mineira de Enfermagem. 2010; 14(1): 88-95.

31. SCUSSIATO LA, et al. Perfil epidemiológico dos acidentes de trabalho graves no Estado do Paraná, Brasil, 2007 a 2010. Epidemiologia e Serviços de Saúde. 2013; 22(4): 621-630.

32. SILVA AR, ARAÚJO TM. Acidentes de trabalho graves no estado da Bahia no período de 2007 a 2012. Revista Baiana de Saúde Pública. 2017; 40(Supl. 2): 57-69.

33. SOUTO CC, et al. Perfil das vítimas de acidentes de transporte terrestre relacionados ao trabalho em unidades de saúde sentinelas de Pernambuco, 2012-2014. Epidemiologia e Serviços de Saúde. 2016; 25(2): 351-361. 